\title{
Fast type I interferon response protects astrocytes from flavivirus infection and virus-induced cytopathic effects
}

\author{
Richard Lindqvist ${ }^{1,2}$, Filip Mundt ${ }^{3}$, Jonathan D. Gilthorpe ${ }^{4}$, Silke Wölfel ${ }^{5}$, Nelson O. Gekara ${ }^{6}$, Andrea Kröger ${ }^{7,8}$ \\ and Anna K. Överby ${ }^{1,2^{*}}$
}

\begin{abstract}
Background: Neurotropic flaviviruses such as tick-borne encephalitis virus (TBEV), Japanese encephalitis virus (JEV), West Nile virus (WNV), and Zika virus (ZIKV) are causative agents of severe brain-related diseases including meningitis, encephalitis, and microcephaly. We have previously shown that local type I interferon response within the central nervous system (CNS) is involved in the protection of mice against tick-borne flavivirus infection. However, the cells responsible for mounting this protective response are not defined.

Methods: Primary astrocytes were isolated from wild-type (WT) and interferon alpha receptor knock out (IFNAR ${ }^{-/-}$) mice and infected with neurotropic flaviviruses. Viral replication and spread, IFN induction and response, and cellular viability were analyzed. Transcriptional levels in primary astrocytes treated with interferon or supernatant from virus-infected cells were analyzed by RNA sequencing and evaluated by different bioinformatics tools.

Results: Here, we show that astrocytes control viral replication of different TBEV strains, JEV, WNV, and ZIKV. In contrast to fibroblast, astrocytes mount a rapid interferon response and restrict viral spread. Furthermore, basal expression levels of key interferon-stimulated genes are high in astrocytes compared to mouse embryonic fibroblasts. Bioinformatic analysis of RNA-sequencing data reveals that astrocytes have established a basal antiviral state which contributes to the rapid viral recognition and upregulation of interferons. The most highly upregulated pathways in neighboring cells were linked to type I interferon response and innate immunity. The restriction in viral growth was dependent on interferon signaling, since loss of the interferon receptor, or its blockade in wild-type cells, resulted in high viral replication and virus-induced cytopathic effects. Astrocyte supernatant from TBEV-infected cells can restrict TBEV growth in astrocytes already $6 \mathrm{~h}$ post infection, the effect on neurons is highly reinforced, and astrocyte supernatant from $3 \mathrm{~h}$ post infection is already protective.
\end{abstract}

Conclusions: These findings suggest that the combination of an intrinsic constitutive antiviral response and the fast induction of type I IFN production by astrocytes play an important role in self-protection of astrocytes and suppression of flavivirus replication in the CNS.

Keywords: Astrocytes, Interferon, TBEV, Flavivirus, Viperin

\footnotetext{
* Correspondence: anna.overby@umu.se

'Department of Clinical Microbiology, Virology, Umeå University, 90185

Umeå, Sweden

${ }^{2}$ The Laboratory for Molecular Infection Medicine Sweden (MIMS), 90187

Umeå, Sweden

Full list of author information is available at the end of the article
} 


\section{Background}

The genus Flavivirus belonging to the family Flaviviridae include important pathogens causing severe human disease including meningitis, encephalitis, hemorrhagic fevers, and microcephaly. The most significant neurotropic flaviviruses are arthropod-borne tick-borne encephalitis virus (TBEV), West Nile virus (WNV), Japanese encephalitis virus (JEV), and Zika virus (ZIKV). TBEV is transmitted by Ixodes ticks, whereas WNV, JEV, and ZIKV are transmitted via mosquitos. No treatments are available for any of these viral infections, and patients are dependent on innate and adaptive parts of the host immune response to fight infections [1-6].

The innate immune system presents a first line of defense against viral infections, for which type I interferons (IFNs) are particularly important. After flavivirus infection, double-stranded RNA (dsRNA) is produced as an intermediate during viral replication. This is sensed as a danger signal in the infected cell by pattern recognition receptors (PRRs) and a signaling cascade is initiated, which leads to the upregulation of IFNs [7, 8]. IFNs are powerful cytokines that mediate antiviral effects via both autocrine and paracrine signaling mechanisms via the IFN alpha receptor (IFNAR). Binding to IFNAR activates the downstream kinases Janus kinase 1 and tyrosine kinase 2, which phosphorylate signal transducer and activator of transcription-1 and transcription-2 (STAT1, STAT2). Together with interferon regulatory factor-9 (IRF9), these form a signaling complex referred to as IFN-stimulated gene factor-3 (ISGF3). ISGF3 translocates to the nucleus and activates the transcription of a large number of interferon-stimulated genes (ISGs) by binding to the interferon response elements. ISGs can inhibit almost every step of a viral life cycle $[9,10]$.

In mice, the type I IFN response is essential for protection against TBEV, JEV, WNV, and ZIKV infections [11-15]. The CNS has been considered as an immune-privileged tissue; however, recent studies have implicated the importance of intrinsic, innate antiviral responses within the CNS [16-19]. In Langat virus (LGTV, Langat virus, low-virulent member of TBEV serogroup) infection, the local type I IFN response in the CNS has been shown to be critical for the protection of mice against lethal encephalitis [11]. However, the CNS cell type responsible for producing IFN during TBEV infection has not been defined.

While neurons are the main target of neurotropic flaviviruses, other cell types might also become infected and contribute to the resolution of infection [20]. Previous studies have shown that the IFN response and ISG expression in neurons restrict neurotropic flavivirus infection in neurons $[19,21]$; however, not much is known about the role of the IFN response in astrocytes during neurotropic flavivirus infection. Recent studies have shown that astrocytes are important IFN-producing cells in various neurotropic viral infections [18, 22, 23]. Astrocytes are one of the most abundant cell types in the brain and mediate diverse supportive functions including ion homeostasis [24, 25], uptake of glutamate [26], free radical scavenging [27], and immune regulation [28]. In TBEV infection, autopsy studies have revealed astrogliosis in post mortem human brains [20,29], which has been observed for WNV and JEV as well [30,31]. Indeed, astrocytes have been found to be a site of these infections [32]. However, only a few astrocytes were found to be infected in LGTV-infected mice [33]. They resist infection in an interferon-beta promoter stimulator 1 (IPS-1)-dependent manner and show an activated phenotype, indicating their involvement in LGTV clearance. Both rat and human astrocytes have been shown to be infected in vitro with TBEV; however, the number of infected cells never exceeded $20 \%$, and the infection did not affect astrocyte viability [34, 35]. Similar findings have also been observed for other neurotropic flaviviruses [36-39]. Therefore, we set out to investigate how the type I IFN system in primary mouse astrocytes contributes to cell survival and restriction of neurotropic flavivirus growth.

We found that astrocytes respond very quickly after viral infection by upregulation of type I IFNs. This upregulation restricts virus replication and spread in primary cultures and contributes to cell survival. By RNA sequencing (RNASeq), we could show that uninfected astrocytes exist in an active antiviral state, which enables fast recognition and response to viral infection by upregulating important antiviral ISGs and that this antiviral state is dependent on IFNAR expression.

\section{Methods}

Mice

C57BL/6 (wild-type (WT)) mice and IFNAR ${ }^{-1-}$ mice on C57BL/6 background were bred at Umeå Transgene Facility.

\section{Isolation of astrocytes and neurons}

The mice were sacrificed between postnatal day 1 and 4 for astrocyte isolation. Cerebral cortices were isolated, and cells were seeded in poly-D-lysine (Sigma) coated T75 tissue culture flasks as previously described [40]. Monolayers of astrocytes were shaken at $200 \mathrm{rpm}$ for $1 \mathrm{~h}$ to remove microglia and oligodendrocyte precursors before seeding for experiments. Primary cortical neurons were derived from cerebral cortices of embryonic day 17 mice. The cortices were isolated, and cells were seeded in Dulbecco's modified Eagle's medium (DMEM, Sigma D5648-10L) containing $10 \%$ heat-inactivated foetal bovine serum (FBS, Gibco) and $0.1 \mathrm{U} / \mathrm{mL}$ penicillin and $0.1 \mu \mathrm{g} / \mathrm{mL}$ streptomycin (Gibco) in poly-D-lysine (Sigma) coated 96-well plates as previously described [41]. After 
$3 \mathrm{~h}$, the medium was replaced with Neurobasal medium (Gibco) containing B27 (Gibco), $0.1 \mathrm{U} / \mathrm{mL}$ penicillin, $0.1 \mu \mathrm{g} / \mathrm{mL}$ streptomycin, and $2 \mathrm{mM} \mathrm{L}$-glutamine (Gibco). The neurons were infected at day 7 post seeding.

\section{Viruses and cells}

VeroB4 cells were cultured in medium 199/EBSS (HyClone) containing $10 \% \mathrm{FBS}, 0.1 \mathrm{U} / \mathrm{mL}$ penicillin, and $0.1 \mu \mathrm{g} / \mathrm{mL}$ streptomycin (Gibco). Mouse embryonic fibroblasts (MEFs) were grown supplemented with $10 \%$ FBS, $50 \mu \mathrm{M} \beta$-mercaptoethanol (AppliChem), and $2 \mu \mathrm{g} /$ $\mathrm{mL}$ tetracycline DMEM (Sigma). TBEV strains Hypr 71 (isolated in 1953 from blood of a patient in the Czech Republic), Aina (isolated in 1963 in Irkutsk from the blood of a patient), and Sofjin (isolated in 1937 from patient in Russia and showed $99 \%$ sequence identity to strains Sofjin-Chumakov and SofjinKSY with BLAST) were a kind gift of G. Dobler (the Bundeswehr Institute of Microbiology, Munich, Germany). JEV (Nakayama strain) was a kind gift of S. Vene (Folkhälsoinstitutet, Stockholm, Sweden). WNV (isolated in 2003 in Israel WNV_0304h_ISR00, passage number 5) is a kind gift of S. Vene. Stocks were generated in VeroB4 cells. Vesicular stomatitis virus (VSV)-eGFP was propagated in VeroB4 cells. ZIKV MR 766 (isolated in 1947 in Zika Forest, Uganda) was propagated in VeroB4 cells and originally provided by Robert Shope (Yale Arbovirus Research Unit, New Haven, CT, USA) as a reference strain to Jürgen Pilaski (University of Düsseldorf, Germany), who kindly transferred the strain to the Bundeswehr Institute of Microbiology, Munich, Germany. TBEV strains, JEV, and ZIKV are cell culture-adapted reference strains with an unknown passage history. Cell monolayers were infected with virus for $1 \mathrm{~h}$ at $37^{\circ} \mathrm{C}$. The virus inoculum was then removed and replaced with DMEM supplemented with $2 \%$ FBS, $0.1 \mathrm{U} / \mathrm{mL}$ penicillin, and $0.1 \mu \mathrm{g} / \mathrm{mL}$ streptomycin. Viral titers were determined by focus forming assay as previously described [42].

\section{RNA isolation and qPCR}

Total RNA was isolated at the indicated time points using Nucleospin RNA II kit (Macherey-Nagel), and cDNA was synthesized from 200 to 600 ng RNA as previously described [42]. mRNA expression of GAPDH, IFN $\beta$, IFN $\alpha 2$, viperin and tripartite motif $79 \alpha$ (TRIM79 $\alpha$ ) were detected by QuantiTect primer assay (Qiagen) and the KAPA SYBR FAST qPCR kit (KAPA Biosystems) using a StepOnePlus fast real-time PCR system (Applied Biosystems). TBEV RNA was quantified using primers previously described [43] and the KAPA PROBE FAST qPCR kit (KAPA Biosystems). Gene expression was normalized to the endogenous GAPDH expression.

\section{Viral spread assay and immunofluorescence}

Cells were grown on 96-well plates (Greiner CELLSTAR ${ }^{\circ}$, fixed in $4 \%$ formaldehyde and permeabilized in PBS containing $0.5 \%$ Triton X-100 and $20 \mathrm{mM}$ glycine. The cells were stained with primary antibodies; a mouse monoclonal TBEV anti-E antibody (Hypr, 1493 1:1000 [44], Sofjin and Aina, 1786 1:1000 [44]), flavivirus anti-E antibody (JEV, WNV, and ZIKV, HB112 1:1000, ATCC D1-4G2-4-15 [45]), and rabbit polyclonal anti-GFAP antibody (1:1000 Abcam, ab7260 [46]). Secondary antibodies were as follows (Thermo Fisher Scientific); donkey anti-rabbit Alexa Fluor 488 (A21206), donkey anti-mouse Alexa Fluor 555 (A31570), and goat anti-mouse Alexa Fluor 647 (A21236) which were diluted 1:500. Nuclear counterstaining was performed using DAPI (Life Technologies, D1306) $1 \mu \mathrm{g} /$ $\mathrm{mL}$. Viral spread was quantified using a TROPHOS Plate RUNNER HD ${ }^{\circ}$ (TROPHOS SA, Marseille, France). Images of immunofluorescence staining were acquired under a Zeiss Axiovert 25 microscope using an infinity3 luminera ${ }^{\circ}$ camera.

\section{IFN bioassay}

Supernatants from virus-infected astrocytes were inactivated using $\beta$-propiolactone ( $\beta$-PL) (Acros Organics, 269040050 ), diluted in water to $0.96 \%$ and used at a final concentration of $0.05 \%$, incubated at $4{ }^{\circ} \mathrm{C}$ for $16 \mathrm{~h}$ followed by $2 \mathrm{~h}$ incubation at $37^{\circ} \mathrm{C}$ for hydrolysis, performed in plates (VWR, 734-2325) to avoid acidification of the samples $[47,48]$. The cells (MEFs, astrocytes, or neurons) were seeded in 96-well plates (Greiner CELLSTAR ${ }^{\circ}$ ) and treated with $\beta$-PL-inactivated supernatants or serially diluted IFNoB/D [49] (a kind gift of Peter Stäheli, Virology, University of Freiburg, Freiburg, Germany). The cells were infected 24 h post treatment with VSV-eGFP (multiplicity of infection (MOI) 0.01) (MEFs) or TBEV (astrocytes or neurons) and fixed with $4 \%$ formaldehyde at $16 \mathrm{~h}$ post infection (hpi). The number of infected cells was determined using a TROPHOS Plate RUNNER HD', and the IFN levels were quantified using a standard curve (calculated from 1 , $5,10,50,100$, and $500 \mathrm{U}$ of IFNaB/D).

\section{Western blot}

The cells were lyzed, proteins were separated, and Western blot was performed as previously described [50]. The primary antibodies directed against TBEV E (1493), actin (rabbit polyclonal anti-actin, 1:2500, Sigma [50]), and viperin (mouse monoclonal anti-viperin, 1:500 Abcam [50]) were used.

\section{RNASeq}

Primary WT and $\mathrm{IFNAR}^{-/-}$astrocytes were seeded at 200,000 cells per 12-well. Upon reaching confluence, WT cells were either stimulated with $\beta$-PL-inactivated supernatant from WT astrocytes (24 hpi), $5000 \mathrm{U}$ IFNaB/D [49], 
or left untreated. Six hours after stimulation, total RNA was isolated using Nucleospin RNA II kit (MachereyNagel) according to the manufacturers' instructions. One thousand to three thousand nanograms of RNA was sent to GATC Biotech (Konstanz, Germany) for RNAseq (InView $^{\mathrm{TM}}$ Transcriptome Explore).

\section{Bioinformatics}

For analyses of the RNASeq data, all treatments were normalized to their respective control and gene names were converted to human HGNC gene names before bioinformatical analyses were performed. Ingenuity Pathway Analysis (IPA; Ingenuity Systems, http:// www.ingenuity.com/) was used to analyze activated pathways and predicted upstream activators as previously described [51, 52]. In brief, activation of $Z$ values were used to determine activation of pathway or regulators $(-2 \geq Z$, significant inhibition; $2 \leq Z$, significant activation), $p$ values were calculated using right-tailed Fisher's exact representing the significance for the overlap between dataset and pathway or regulator. Gene set enrichment analysis (GSEA; http://www.broadinstitute. org/gsea) was performed to evaluate functional gene sets that are associated with each of the treatments compared to controls. The fold-change regulation of the genes after treatment was used to perform pre-ranked GSEA. The BIOCARTA, KEGG, and REACTOME databases were queried; $p$ values were estimated by 100 permutations and a null-distribution of the enrichment scores. $P$ values were adjusted using the BenjaminiHochberg method. A normalized enrichment score (NES) was computed by mean normalization [53]. The Connectivity Map database (http://www.lincscloud.org/; [54]) was used to analyze upstream regulators based on regulated genes after IFN $\alpha \mathrm{B} / \mathrm{D}$ or supernatant treatment. The top 50 expressed genes and top 50 downregulated genes were selected from, each treatment, per analysis, and a connectivity score $>90$ or $<-90$ was considered significant.

\section{Blocking of IFNAR receptor}

Cells were incubated with DMEM, 10 \% heat-inactivated FBS, and $2 \mathrm{mM}$ glutamine with MAR1-5A3 antibody (Affymetrix eBioscience, 16-5945-85 [19]) or IgG1 $\mathrm{k}$ isotype control (eBioscience, 14-4714-85) at a concentration of $10 \mu \mathrm{g} / \mathrm{mL}$ for $2 \mathrm{~h}$ at $37^{\circ} \mathrm{C}$. After $2 \mathrm{~h}$, the antibodies were removed, and cells were infected with TBEV for $1 \mathrm{~h}$. Following removal of the viral infection inoculum, the medium containing $10 \mu \mathrm{g} / \mathrm{mL}$ antibody was returned to the cells.

\section{Resazurin viability test}

Three hours before the indicated time point, the cells were treated with $40 \mu \mathrm{M}$ resazurin; after $3 \mathrm{~h}$ of incubation, fluorescent signal was quantified using a plate reader (Paradigm, Beckman Coulter).

\section{Statistical analyses}

Data from quantitative reverse transcription PCR (qPCR), bioassay, focus forming assays, and viral spread assays were analyzed with unpaired $t$ test using GraphPad Prism software. Statistical analyses of RNASeq data were performed by GATC Biotech using Cufflinks $[55,56]$.

\section{Results \\ The type I IFN response limits TBEV replication in astrocytes}

Astrocytes are the most abundant glial cell type in the brain and an important source of type I IFN during various neurotropic viral infections $[18,22,23]$. To investigate the importance of type I IFNs for astrocyte function in neurotropic flavivirus infection, primary astrocytes were isolated from wild-type (WT) and IFNAR ${ }^{-1-}$ mice. These were infected with TBEV strain Hypr at a MOI of 0.1 and analyzed by immunofluorescence (Fig. 1a). Both WT and IFNAR $^{-1-}$ infected astrocytes expressed the characteristic marker glial fibrillary acidic protein (GFAP) and were found positive for TBEV E (Fig. 1a). Viral replication was analyzed over time, and low viral titers and replication were observed in WT astrocytes. In contrast, TBEV titers and RNA replication increased dramatically in IFNAR ${ }^{-/-}$astrocytes over time (Fig. 1b, c). Infection was also analyzed in MEFs where an unrestricted growth profile was observed in both WT and IFNAR $^{-/-}$cells (Fig. 1d, e). Our results indicate that IFNAR restricts TBEV replication in a cell type-dependent manner.

\section{IFNAR restricts neurotropic flavivirus spread in astrocytes}

The type I IFN response could limit viral replication by different mechanisms: (i) restricting the spread of progeny viruses to neighboring cells, (ii) reducing overall viral replication, or (iii) both. To investigate this, WT and IFNAR $^{-/-}$astrocytes and MEFs were infected by TBEV at a MOI of 0.1, stained by immunofluorescence, and the numbers of infected cells were quantified (Fig. 2a shows a representative picture of infected wells). Even though the initial infections (24 hpi) in WT and IFNAR ${ }^{-/}$ astrocytes were comparable, the outcome of infection differed dramatically. Infection of WT astrocytes was low and never exceeded $20 \%$; however, infection of IFNAR ${ }^{-1-}$ astrocytes was significantly higher at the late time points, reaching an average of $69 \%$ at 72 hpi (Fig. 2b). In MEFs, IFNAR expression failed to restrict TBEV spread, and no difference were observed between WT and IFNAR ${ }^{-1-}$ cells (data not shown). These results suggest that IFNAR restricts the spread of TBEV in astrocytes. 


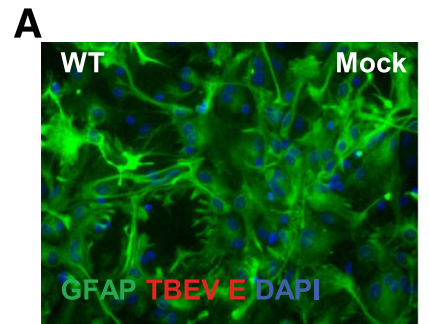

B

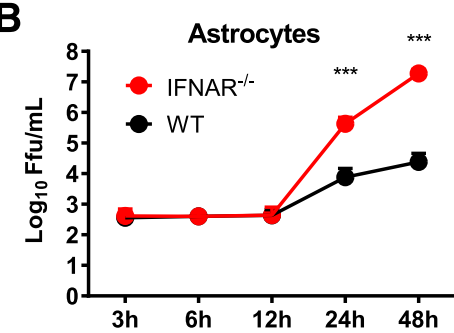

D

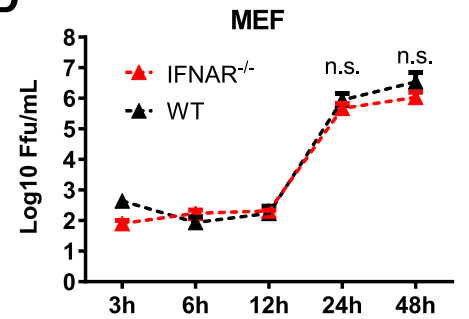

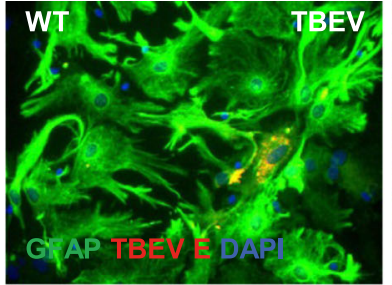

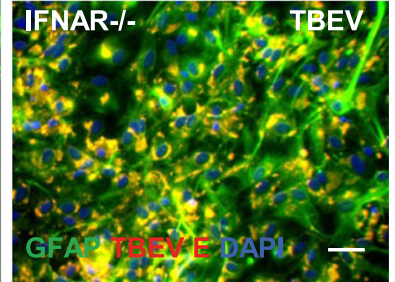

C

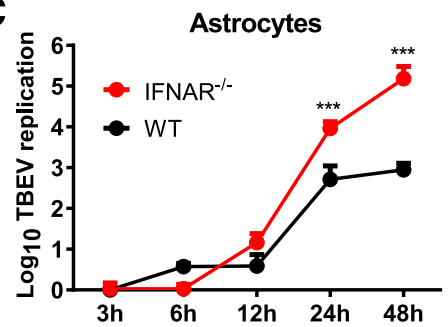

E

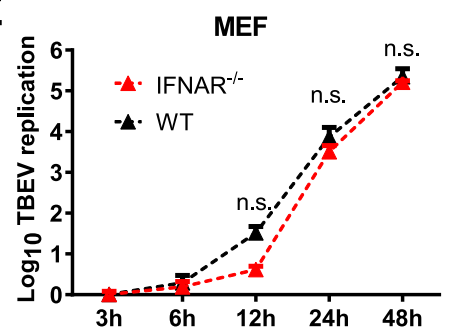

Fig. 1 IFNAR restricts TBEV replication in astrocytes. Primary astrocytes were isolated from WT and IFNAR ${ }^{-1-}$ mice and infected with Hypr MOI 0.1. a Cell were stained 72 hpi with GFAP (astrocytes), TBEV E protein, and DAPI. Scale bar $193 \mu$ m. Primary astrocytes (b, c) and MEF (d, e) were infected with TBEV (MOI 0.1), and viral growth was determined over time by focus forming assay (b, d) and qPCR (c, e). Data are cumulative from at least two independent experiments performed in triplicates $(n=6)$. Asterisks indicate data were statistically significant: ${ }^{* * *} p<0.0001$

TBEV can be categorized into European, Siberian, and Far Eastern subtypes according to phylogenetic differences. The Siberian and Far Eastern subtypes have been associated with more severe disease compared to the European subtype $[57,58]$. Increased pathogenicity could be due to several factors, e.g., ability to interfere with the type I IFN response. Therefore, viral growth of two reference strains; the Siberian Aina and Far Eastern Sofjin, was analyzed in WT and IFNAR ${ }^{-/-}$astrocytes (Fig. 2c, d). Similar to the European subtype of TBEV, an interferon response also restricted the spread of Siberian and Far Eastern TBEV subtypes (Fig. 2c, d). Although the type I IFN response plays an important role in mosquito-borne neurotropic flavivirus (WNV, JEV, and ZIKV) infections in vivo $[12-14,59,60]$, the specific role of IFNAR signaling in restricting viral growth in astrocytes is unknown. WT and IFNAR ${ }^{-/-}$astrocytes were infected with JEV, WNV, and ZIKV, and the numbers of infected cells were determined (Fig. 2e-g). For all three mosquito-borne viruses, the IFN response was required for the restriction of viral spread in astrocytes. Our data shows that the type I IFN response in astrocytes efficiently restricts the spread of both tick- and mosquito-borne neurotropic flaviviruses.

\section{Astrocytes induce a rapid IFN response upon TBEV infection}

Previous studies have indicated the importance of astrocyte-produced IFN in various viral infections $[18,22,23]$. The observed differences in the impact of IFNAR on astrocytes and MEFs could either be explained by the ability to respond to, or the capacity to induce, IFN following infection. To determine the impact of the type I IFN response in the overall antiviral response, astrocytes and MEFs were pretreated with IFN $\alpha B / D$ [49] Sixteen hours before TBEV infection (Fig. 3a, b). TBEV growth was inhibited to a similar extent showing that both cell-types were able to mount a strong antiviral response upon IFN treatment. Thus, the ability to respond to IFN (Fig. 3a, b) could not explain the lack of control of viral replication in IFNAR $^{-/-}$MEFs (Fig. 1d, e). To test whether astrocytes and MEFs differ in their capability to induce type I IFNs after infection, IFN $\beta$ and IFN $\alpha 2$ mRNAs were quantified by realtime qPCR (Fig. 3c, d). Interestingly, the IFN responses in the two cell types were quite different. Whereas astrocytes induced IFN mRNA after $6 \mathrm{~h}$, the response in MEFs was delayed and IFN $\beta$ and IFN $\alpha 2$ mRNAs were detected only after 24 or 48 hpi, respectively. Next, a VSV-GFP-based 


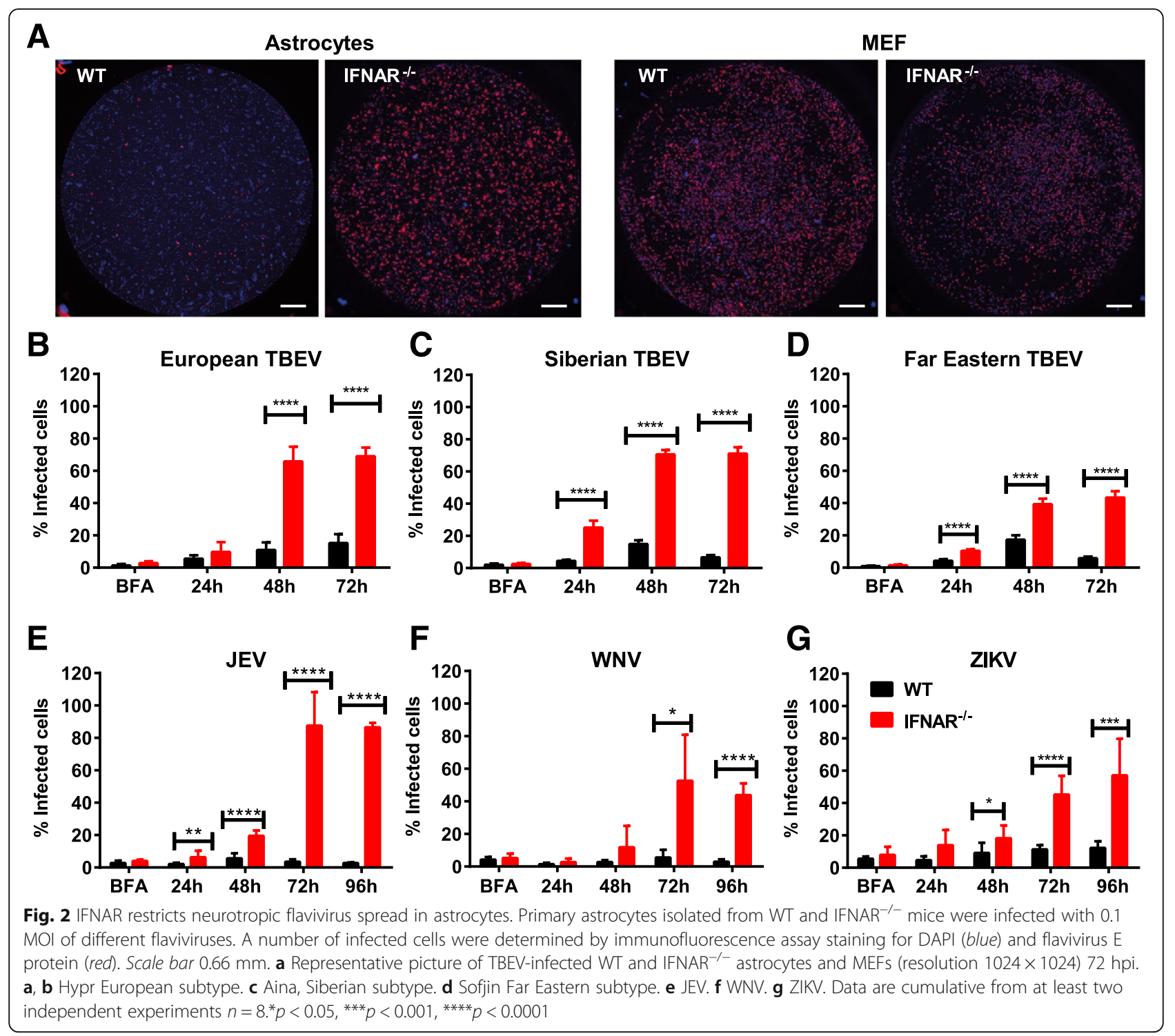

bioassay on MEFs was applied to quantify secreted IFN, and astrocytes were found to secrete higher levels of IFNs 24 hpi compared to MEFs (Fig. 3e). These data indicate that astrocytes react to viral infection by the induction of IFNs much more rapidly than MEFs.

In order to define the time point after infection at which astrocytes initiate an antiviral response and limit viral spread in astrocytes, a TBEV-based bioassay on astrocytes was developed. Pretreated astrocytes were infected, and the numbers of infected cells were quantified at 48 hpi (Fig. 3f). The supernatants showed an inhibitory effect as early as $6 \mathrm{hpi}$ on WT, but not on IFNAR $^{-/-}$cells, suggesting a very early type I IFNmediated antiviral effect.

The antiviral effect of the supernatant was further investigated using $\mathrm{GPCR}$ as a more sensitive method of quantifying viral replication. Sixteen hours before TBEV infection, the cells were treated with either supernatant of 24-h TBEV-infected cells or IFN $\alpha B / D$. The cells were infected with TBEV, and total RNA was extracted 48 hpi to detect TBEV RNA (Fig. 3g). Pretreatment with supernatant of infected cells and $5000 \mathrm{U}$ IFN $\alpha \mathrm{B} / \mathrm{D}$ had a similar inhibitory effect on TBEV replication in WT astrocytes. However, no difference in viral replication was detected in IFNAR $^{-/-}$astrocytes treated with or without supernatant, indicating a type I IFN-dependent antiviral effect. These results further suggest that the rapid type I IFN response is responsible for limiting viral infection and replication in astrocytes.

IFNAR mediates antiviral preparedness in astrocytes Our data indicate that WT astrocytes are quite resistant to viral infection and that the main effectors in the supernatant from virus-infected astrocytes are type I 

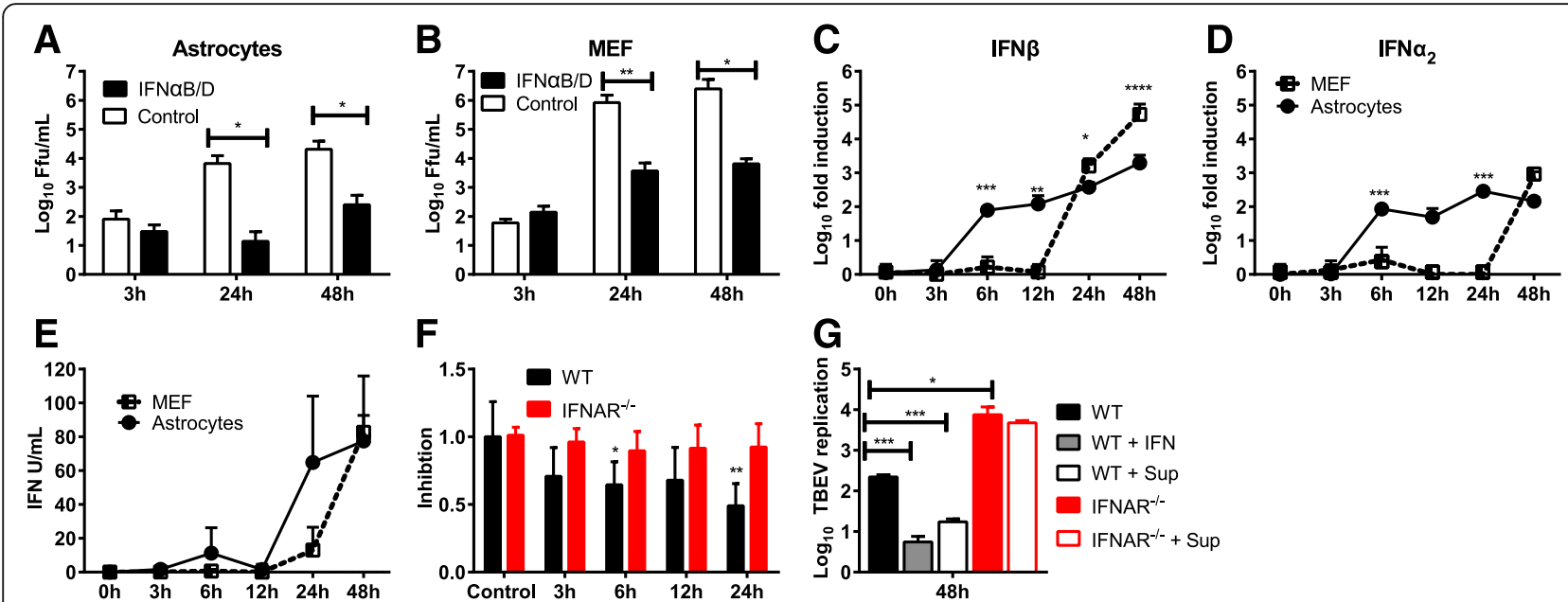

Fig. 3 Astrocytes induce a fast type I IFN response upon TBEV infection which restricts virus replication. Primary WT Astrocytes (a) and MEFs (b) were pretreated with $5000 \mathrm{U}$ IFNaB/D for $16 \mathrm{~h}$ before infection with TBEV MOI 0.1. Viral titers were determined by focus forming assay at indicated time points $(n=6)$. Primary astrocytes and MEFs were infected with TBEV using MOI 0.1. Expression levels of IFN- $\beta(\mathbf{c})$ and IFNa $2(\mathbf{d}) \mathrm{were}$ determined by qPCR $(n=9)$. Supernatants were collected at the indicated time points, and antiviral activity was determined by VSV-GFP bioassay on MEFs $(\mathbf{e}, n=6)$ or TBEV-based bioassay on WT and IFNAR ${ }^{-/-}$astrocytes $(\mathbf{f}, n=12) . \mathbf{g ~ W T}$ and IFNAR ${ }^{-/-}$astrocytes were treated with either $5000 \mathrm{U}$ IFNaB/D or virus-inactivated supernatant from WT astrocytes $24 \mathrm{hpi}(n=6)$. TBEV replication was quantified using qPCR and normalized to input viral RNA. Mean values and standard deviations from three independent experiments are shown. ${ }^{*} p<0.05 ;{ }^{* *} p<0.01 ;{ }^{* *} p<0.001$

IFNs. To characterize these responses in more detail, we analyzed transcriptional regulation by RNAseq. Gene expression levels from uninfected IFNAR ${ }^{-/-}$astrocytes and IFNaB/D-treated, or supernatant-treated, WT astrocytes were compared to untreated WT astrocytes. Fold-change values of at least twofold over the uninfected control and a $q$ value of $<0.05$ were considered indicative of upor downregulation. Our data yielded 732 upregulated transcripts while 944 were found to be downregulated in IFNAR $^{-1-}$ astrocytes. Treatment of primary astrocytes with IFN $\alpha$ B/D or supernatant led to 634 or 1092 upregulated and 149 or 423 downregulated genes, respectively (Additional file 1: Table S1).

The majority of all significantly regulated genes showed a unique pattern of regulation for each sample (Fig. $4 \mathrm{a}-\mathrm{c})$. Looking more closely at the differences between astrocytes treated with supernatant or IFNaB/D, relatively few genes were downregulated, and we detected 257 genes upregulated in both samples, out of total 894 upregulated genes in the supernatant and 550 in IFNaB/D (Fig. 4b, c). This indicated that although the main transcriptional response is type I IFN-dependent and overlaps with IFN $\alpha B / D$ treatment, virus infection induces numerous other IFN-independent responses in neighboring, uninfected astrocytes.

Only 112 transcripts were found to be significantly regulated between all three samples compared to WT (Fig. 4a). However, they showed a very different expression profile (Fig. 4d). Interestingly, IFNAR-deficient astrocytes showed lower levels of PRR, innate immune signaling, transcription factors, ISGs, and adaptive immune response compared to untreated WT astrocytes, whereas, IFN $\alpha B / D$ - and supernatant-treated WT astrocytes showed an upregulated profile (Fig. 4d, Additional file 1: Table S2).

\section{WT astrocytes show higher expression and rapid} induction of antiviral ISGs after TBEV infection The susceptibility of IFNAR ${ }^{-/-}$astrocytes to flavivirus infection might be due to lowered basal expression of antiviral ISGs, such as viperin. Together with TRIM79 $\alpha$, viperin has recently been identified as an inhibitor of TBEV in vitro [50,61]. Viperin has also been shown to have antiviral activity against WNV [62]. Viperin and TRIM79 $\alpha$ mRNAs were quantified over time by qPCR in WT and IFNAR ${ }^{-/-}$astrocytes and WT MEFs (Fig. 5a, b). ISGs were rapidly induced in WT astrocytes at early time points whereas induction was delayed in IFNAR ${ }^{-/-}$ astrocytes and WT MEFs. The basal levels of viperin and TRIM79 $\alpha$ were higher in WT astrocytes compared to WT MEFs and IFNAR ${ }^{-/-}$astrocytes (Fig. 5b) and might contribute to limit initial viral infection. Viperin protein was also strongly induced in WT astrocytes as early as 6 hpi whereas corresponding protein levels were only reached after $24 \mathrm{hpi}$ in IFNAR $^{-/-}$astrocytes (Fig. 5c). Together, these data show that WT astrocytes express higher basal levels of a subset of antiviral genes and rapidly respond upon viral infection to express antiviral protein that can limit viral infection. MEFs and IFNAR $^{-1-}$ astrocytes show lower basal expression of some antiviral ISGs and a delayed IFN response and ISG 


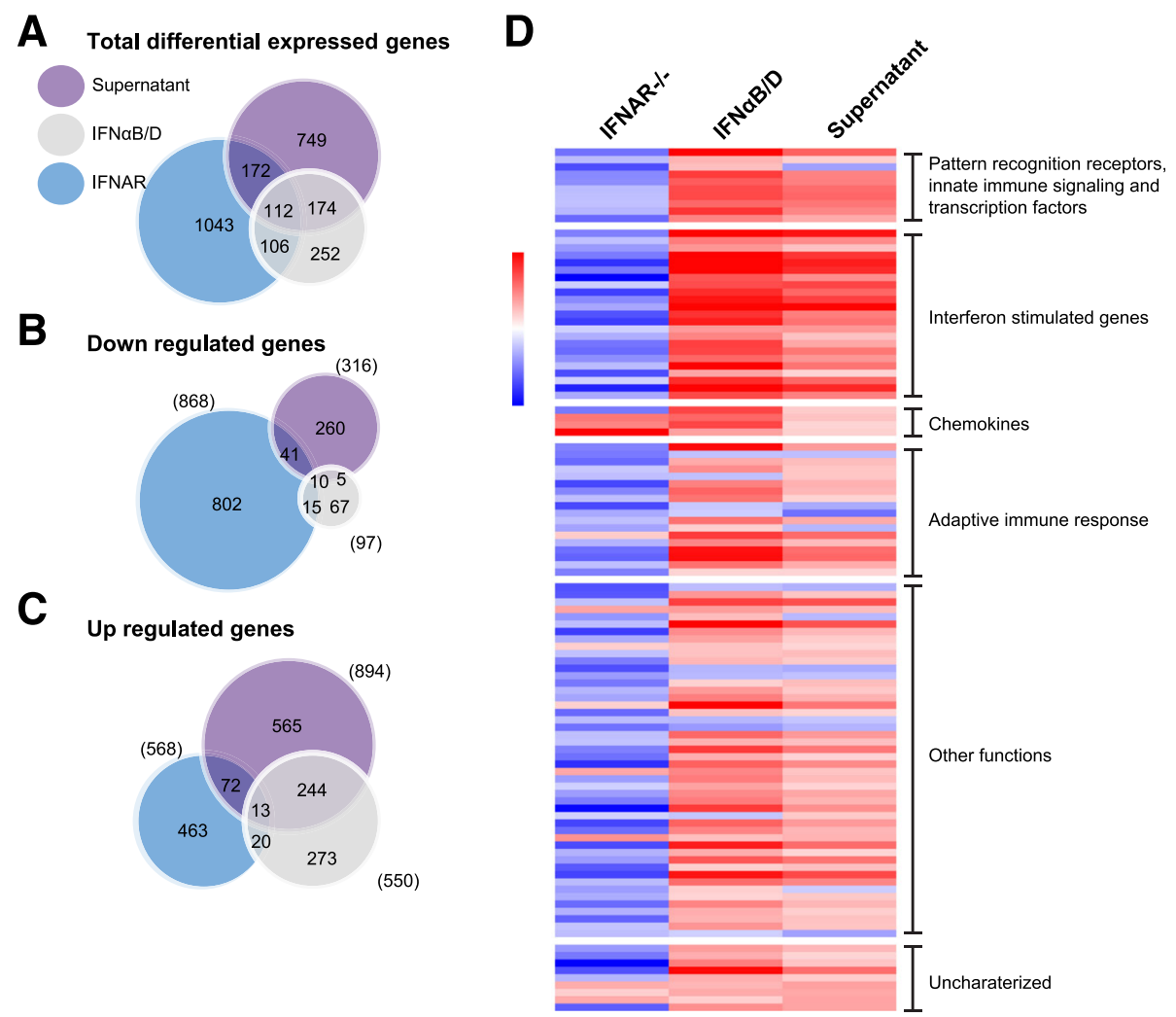

Fig. 4 Astrocytes treated with either supernatant or IFNaB/D mount an antiviral response. Primary astrocytes from WT and IFNAR ${ }^{-/-}$mice were isolated, and WT cells were either mock treated, treated with IFNaB/D, or treated with inactivated supernatant from TBEV-infected astrocytes. Gene expression was analyzed by deep sequencing. Venn diagram of differentially expressed transcripts in IFNAR ${ }^{-1}$, IFNaB/D, and supernatant-treated astrocytes compared to mock-treated WT astrocytes (fold-change \pm 2 , q value $<0.05$ ). Number of totally regulated genes (a), downregulated (b), and upregulated (c). d Overlap of differentially expressed transcripts under all three conditions $(n=112)$; red color indicates an upregulation whereas blue color correlates with a downregulation. Dynamic range in the heat map is 7.16 to $-5.99 \log 2$ fold difference compared to WT untreated astrocytes. Sd standard deviation $(n=3)$

induction which is likely to render them more susceptible to viral infection.

\section{IFN signaling restricts TBEV spread in astrocytes}

Gene set enrichment analysis was used to identify classes of genes overrepresented in the dataset. IFNAR ${ }^{-/-}$cells showed a negative enrichment compared to WT in immune pathways and interferon signaling, while the positively enriched gene sets were below our cut-off adjusted $p$ value $<0.05$ (Fig. 6). Both IFNaB/D and supernatant treatment lead to enrichment in "interferon signaling" and "interferon alpha beta signaling" (Fig. 6). Differentially expressed genes between WT and the three samples were analyzed using IPA to uncover predicted activation of canonical pathways and disease functions. Pathways involved in viral recognition and antiviral signaling as well as functions involved in viability of leukocytes and antiviral and inflammatory responses were found to be downregulated in IFNAR ${ }^{-1-}$ compared to WT astrocytes (Additional file 1: Tables S3 and S4), which can explain why the IFNAR $^{-/-}$cells responded poorly after viral infection. IPA analysis revealed activation of pathways involved in innate immune signaling and viral recognition, antiviral and immune response after treatment with IFN $\alpha B / D$ and supernatant (Additional file 1: Table S3 and S4). To extend our analyses and investigate what might be responsible for these transcriptomic changes, with focus on the difference between the IFNaB/ $\mathrm{D}$ and the supernatant treatment, we performed an IPA upstream analysis, and a Connectivity Map (CMAP) analysis. Both of these analyses evaluate the relationship(s) that might have led to the pattern of transcript regulation that we found after treatment. In Fig. 7a, we have plotted all upstream regulators that are predicted by the IPA analysis to be either activated or inhibited after IFN $\alpha B / D$ or supernatant treatment. Upstream regulators that are predicted to be activated after both treatments include several interferon regulated factors (IRF3, IRF7, STAT1) as well as IFNAR and IFN $\gamma$ as the most activated. Each dot in Fig. 7a represents a regulator e.g., (IFN alpha/beta) with a circle of markers of upregulated transcripts found in the dataset (Fig. 7b). Interestingly, five upstream regulators were 

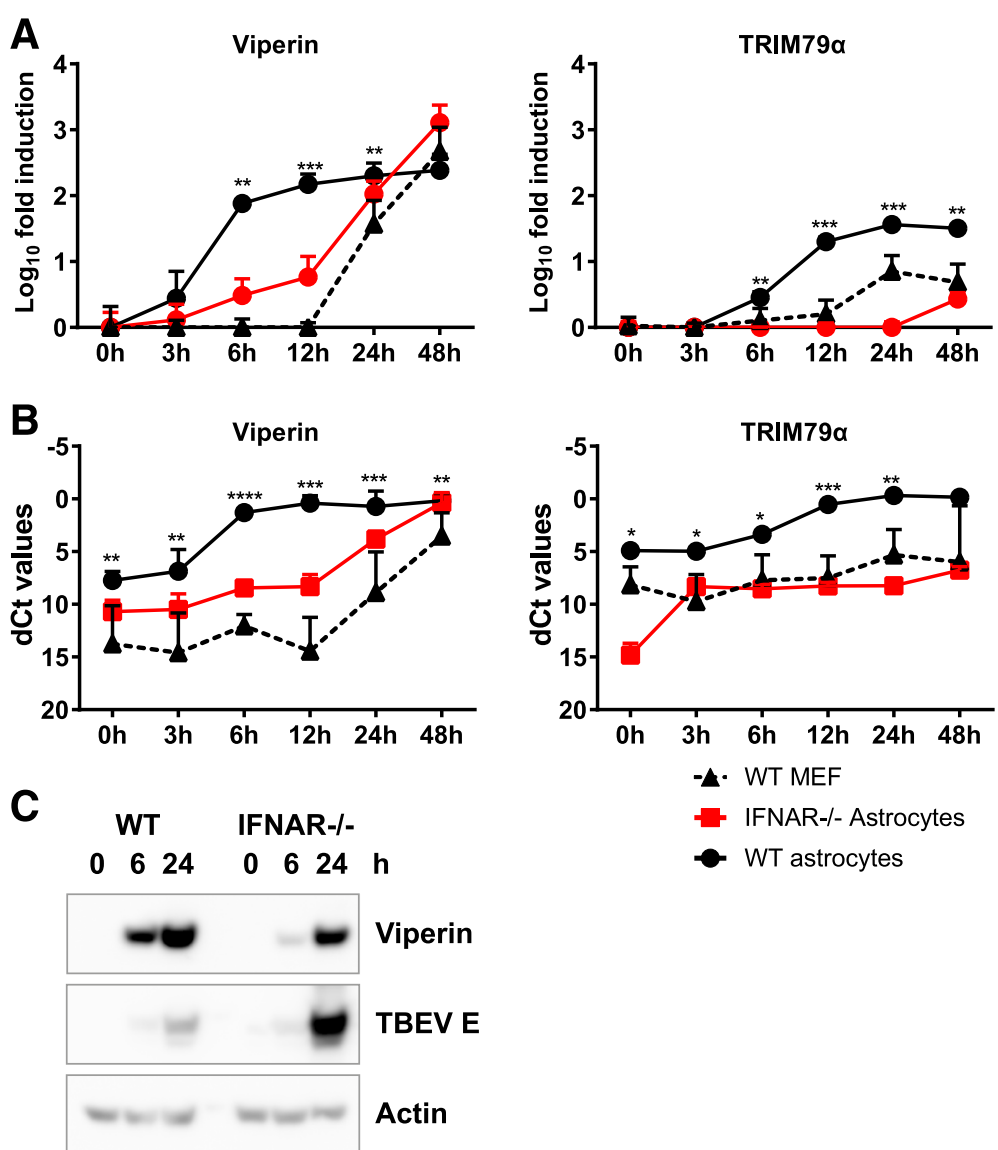

Fig. 5 Astrocytes express higher baseline levels and upregulate ISGs faster after TBEV infection compared to MEFs. Primary astrocytes (WT and IFNAR $^{-1-}$ ) and MEFs were infected with TBEV MOI 0.1, and total cell RNA was extracted at indicated times post infection. Expression levels of viperin and TRIM79a $(\mathbf{a}, \mathbf{b})$ were measured by qPCR analysis, normalized to the cellular GAPDH mRNA. Fold induction over mock (a) and dCT values $(\mathbf{b})$ are depicted. Mean values and standard deviations from three independent experiments performed in triplicates are shown $(n=9)$. Asterisk indicates the significance level between WT astrocytes and MEF. ${ }^{*} p<0.05,{ }^{* *} p<0.01,{ }^{* * *} p<0.001$. Protein levels of viperin, TBEV, and actin was detected in TBEV-infected WT and IFNAR ${ }^{-/}$astrocytes over time (c)

predicted to be activated specifically after supernatant treatment (IL5, CD38, KMT2D, HIF1A, UCP1, and JAK2; Fig. 7a, $y$-axis $>2$ ). The CMAP database is an orthogonal bioinformatics tool that correlates specific geneoverexpression with their associated transcriptomic changes. Our transcriptional changes after IFNaB/D or supernatant treatments were compared to the CMAP database and the connectivity scores are compared to each other in Fig. 7c, and top scoring genes included IFNB1, IFN $\gamma$, and CD40. IFN $\gamma$ came up in both gene set enrichment analyses, IPA and CMAP (Figs. 6, 7a, b), suggesting that TBEV induces IFN $\gamma$ after infection in astrocytes. However, no differences in IFNy mRNA expression levels were detected after infection in astrocytes (data not shown).

With this comprehensive bioinformatics analysis, we can show that the main gene sets enriched after supernatant treatment are IFN signaling gene sets very similar to the IFN $\alpha B / D$ treatment (Fig. 6), overall indicating that IFNs are the main response induced after infection. We show that IFNAR ${ }^{-/-}$astrocytes not only express lower levels of key antiviral ISGs (Fig. 5) but also express lower levels of ISGs, PRRs, innate immune signaling, and transcription factors generally (Fig. $4 \mathrm{~d}$ and Additional file 1: Table S2), which probably contributes to the susceptibility of these cells (Figs. 1 and 2).

Astrocytes resistance to viral infection might be due to high basal expression of antiviral genes, fast response via IFNAR signaling, or both. In order to distinguish between these possibilities, monoclonal antibodies were used to block IFNAR signaling in WT astrocytes [63] (Fig. 8a-c). Interestingly, the numbers of TBEV-infected cells and TBEV replication were increased to levels similar to those measured in IFNAR ${ }^{-/}$cells. This indicated that response to type I IFN is more important for restricting viral growth than the basal expression level of antiviral ISGs. Previous studies have shown that astrocytes are resistant to TBEV-induced cytopathic effects $[34,35]$. We can now show that the viability of WT 


\title{
IFNAR-/-
}

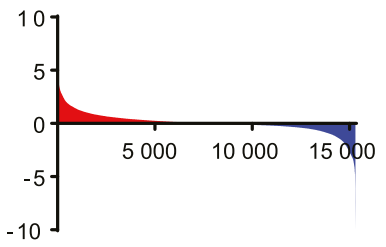

NES pval adj.pval

$-2.12<0.001<0.001$

$-2.03<0.001<0.001$

$-2.03<0.001<0.001$

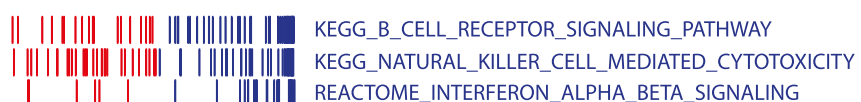

\section{IFNaB/D}

$\begin{array}{ccc}\text { NES } & \text { pval } & \text { adj.pval } \\ 2.25 & <0.001 & <0.001 \\ 2.17 & <0.001 & <0.001 \\ 2.05 & <0.001 & <0.001\end{array}$

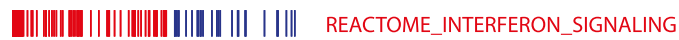
- \|\|$\|\quad|\|| \quad||$ REACTOME_INTERFERON_ALPHA_BETA_SIGNALING $2.05<0.001<0.001$

\section{Supernatant}

$\begin{array}{ccc}\text { NES } & \text { pval } & \text { adj.pval } \\ 2.39 & <0.001 & <0.001 \\ 2.25 & <0.001 & <0.001 \\ 2.15 & <0.001 & <0.001 \\ & & \\ -2.54 & <0.001 & <0.001 \\ -2.49 & <0.001 & <0.001 \\ -2.25 & <0.001 & <0.001\end{array}$

\author{
\|\|\|\|||||||$\quad|| \quad$ REACTOME_INTERFERON_ALPHA_BETA_SIGNALING \\ ||||| ||||||||||||||| | ||||||||||||||||||||||||| REACTOME_INTERFERON_SIGNALING \\ \|\||||||||||||||||| REACTOME_INTERFERON_GAMMA_SIGNALING \\ | |||||||||||| $\mid$ REACTOME_RNA_POL_I_PROMOTER_OPENING \\ | $\|||||||||||||||||||||||||||$ REACTOME_CHROMOSOME_MAINTENANCE \\ | | $\||\||\|\mid\|$ REACTOME_DEPOSITION_OF_NEW_CENPA_CONTAINING_ \\ NUCLEOSOMES_AT_THE_CENTROMERE
}

Fig. 6 Gene set enrichment analysis of transcriptomic changes. The schematic histogram shows a typical expression profile of the transcriptomic changes after treatment ( $y$-axis = fold changes; $x$-axis = one vertical line (stick) represents one transcript; red, up; blue, down). mRNA transcripts (sticks) and gene sets (names) that are high in IFNAR ${ }^{-/-}$astrocytes, IFNaB/D and supernatant-treated WT astrocytes, compared to WT astrocytes, are marked as red. Genes and gene sets that are downregulated or have a negative normalized enrichment score (NES), respectively, compared to WT astrocytes are blue. pval nominal $p$ value, adj.pval Benjamini-Hochberg adjusted $p$ value. An adj.pval <0.05 is regarded significant

astrocytes after TBEV infection is dependent on the type 1 IFN response, since IFNAR ${ }^{-/-}$, as well as WT astrocytes treated with an IFNAR-specific antibody, rendered them susceptible to TBEV-induced cytopathic effect 48 hpi (Fig. 8d).

Neurons are the main target of neurotropic flavivirus infection; however, it is not clear if and how astrocytes influence viral growth in neurons. Therefore, supernatants from infected astrocytes were collected at different time points post infection and inactivated. Primary neurons were treated with supernatants before TBEV infection. The number of infected neurons decreased already when pretreated with supernatants from astrocytes infected for $3 \mathrm{~h}$ (Fig. 8e). These data indicate that astrocytes can mediate a very fast antiviral effect on neurons already $3 \mathrm{~h}$ post infection.

Here, we show that astrocytes are in an antiviral state and respond quickly to flavivirus infection by upregulating type 1 IFNs which limits neurotropic flavivirus spread. Secreted IFNs control viral replication by upregulating several innate immune pathways and induce cell survival. Our data suggest that although neurons seem to represent the main target for neurotropic flavivirus infection in vivo [64], astrocytes are likely to play an important role in responding to infection, amplifying the type I IFN response and limiting viral spread in both astrocytes and neurons.

\section{Discussion}

Previous work has indicated that a local type I IFN response is indispensable for the control of viral replication in the CNS after flavivirus infection [11]. TBEV preferentially replicates in neurons [11, 33], but why astrocytes are less susceptible to TBEV infection remains unclear. In the current study, we showed that astrocytes are initially infected by TBEV but showed strong inhibition of viral spread. Astrocytes showed a higher basal level of ISG expression which enables the cells to rapidly respond with type I IFN production. Thus, astrocytes induce an early and strong antiviral response that limits viral spread in neurons and thereby plays a specific role in the innate antiviral defense in the CNS.

Local type I IFN production is critical to limit viral spread within the CNS [65] whereas CNS deficiency in IFNAR increases the susceptibility of lethal virus infection [13, 17, 18, 66-68]. Using the TBEV model LGTV, we have previously demonstrated the impact of locally produced type I IFN response within the CNS [11]; 

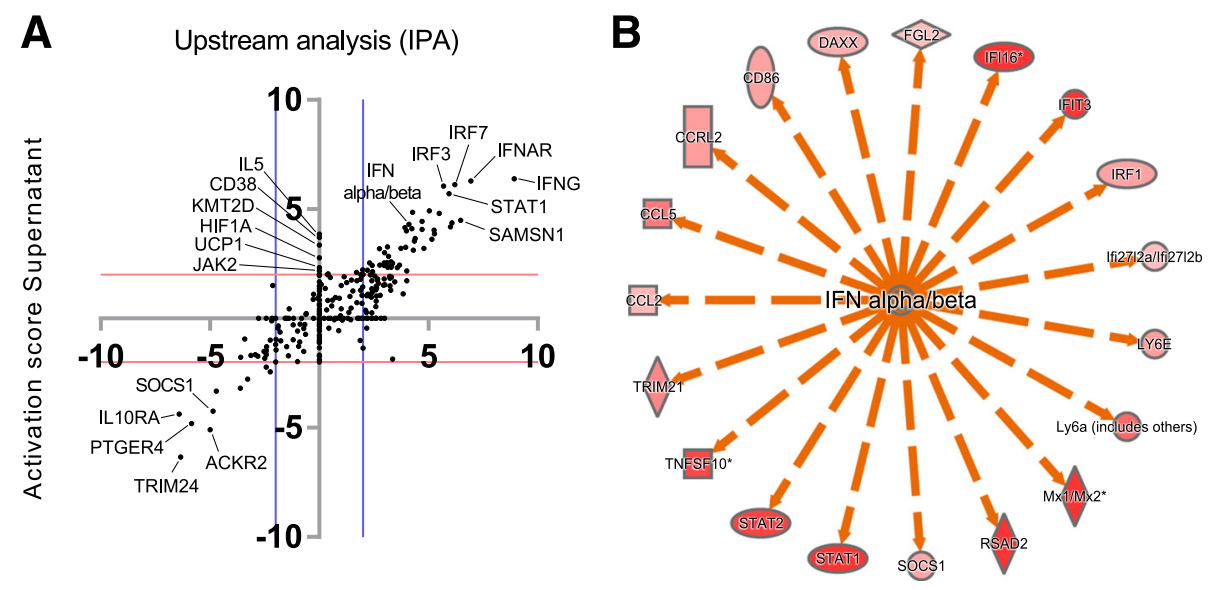

Activation score IFN

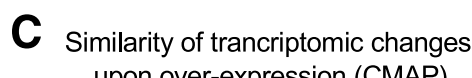
upon over-expression (CMAP)

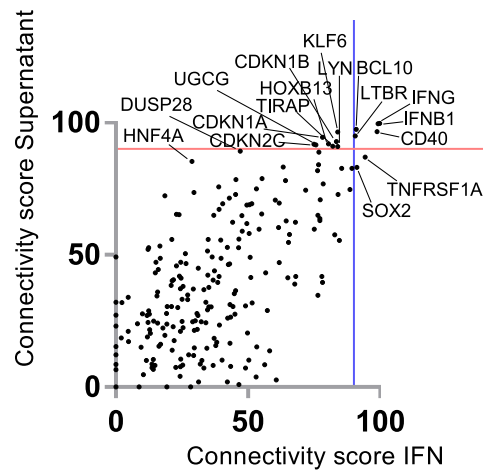

Fig. 7 Upstream activators related to treatment with either supernatant or interferon alpha (IFN). a Scatter blot of ingenuity pathway analysis (IPA) showing upstream regulators that are predicted to be activated ( $>2$ activation score) or inhibited ( $<-2$ activation score) according to experimental and literature findings. $Y$-axis show upstream regulators predicted from supernatant-treated astrocytes, and $x$-axis show upstream regulators predicted from IFNaB/D-treated astrocytes. Five upstream regulators show an idiosyncratic activation specific for the supernatant-treated cells, aligning the $y$-axis and with an activations score of $>2$. b An example of predicted activators by IPA. The circle of markers is upregulated transcripts (red), and the center is a regulator (IFN alpha/beta), predicted to be active based on information (orange arrows) in the IPA knowledge-database. Each black dot in a is representing a similar circle of regulated markers. $\mathbf{c}$ Genes that, when overexpressed, lead to a similar expression pattern as either supernatant treatment ( $y$-axis) or IFNaB/D treatment ( $x$-axis) according to the connectivity map database (CMAP). Genes predicted by both treatments are in the top right corner (e.g., IFNG and IFNB1) while effects exclusive to supernatant treatment are high $(>90)$ on the $y$-axis and low $(<90)$ on the $x$-axis

however, it is not clear which cells produce type I IFN in the CNS during TBEV infection. Astrocytes have been shown to produce an array of innate inflammatory mediators upon stimulation using polyinosinic:polycytidylic acid, lipopolysaccharide, and toll-like receptor (TLR)-7 and TLR-9 agonists [69-72]. Studies have shown that astrocytes are the main producers of type I IFN within the CNS during VSV and La Cross virus infections [18, 23]. Recent studies have shown that TBEV and WNV can infect astrocytes in vitro but fail to spread from cell to cell. However, what prevents viral spread in astrocytes is not known $[34,36]$. Our recent results show that neurons are the main target of LGTV infection; however, a small number of astrocytes also become infected. During infection, astrocytes show an activated phenotype in vivo and more cells become infected after LGTV infection in IPS-1-deficient mice [33], indicating that type I IFN response might contribute to the restricted growth of virus in astrocytes.

In this study, we show that, although primary astrocytes are infected in a comparable manner to MEF initially, viral replication and spread is dramatically inhibited, indicating that astrocytes are abortively infected with neurotropic flavivirus similar to La Crosse virus infections [73]. This phenotype is dependent on IFNAR expression, since viral replication is uncontrolled in IFNAR-deficient astrocytes. Consistent with previous results for WNV, we now show for all three subtypes of TBEV, JEV, and ZIKV that the rapidly produced type I IFN in astrocytes after neurotropic flavivirus infection limits the viral spread and prevent virus-induced killing of the cells. 


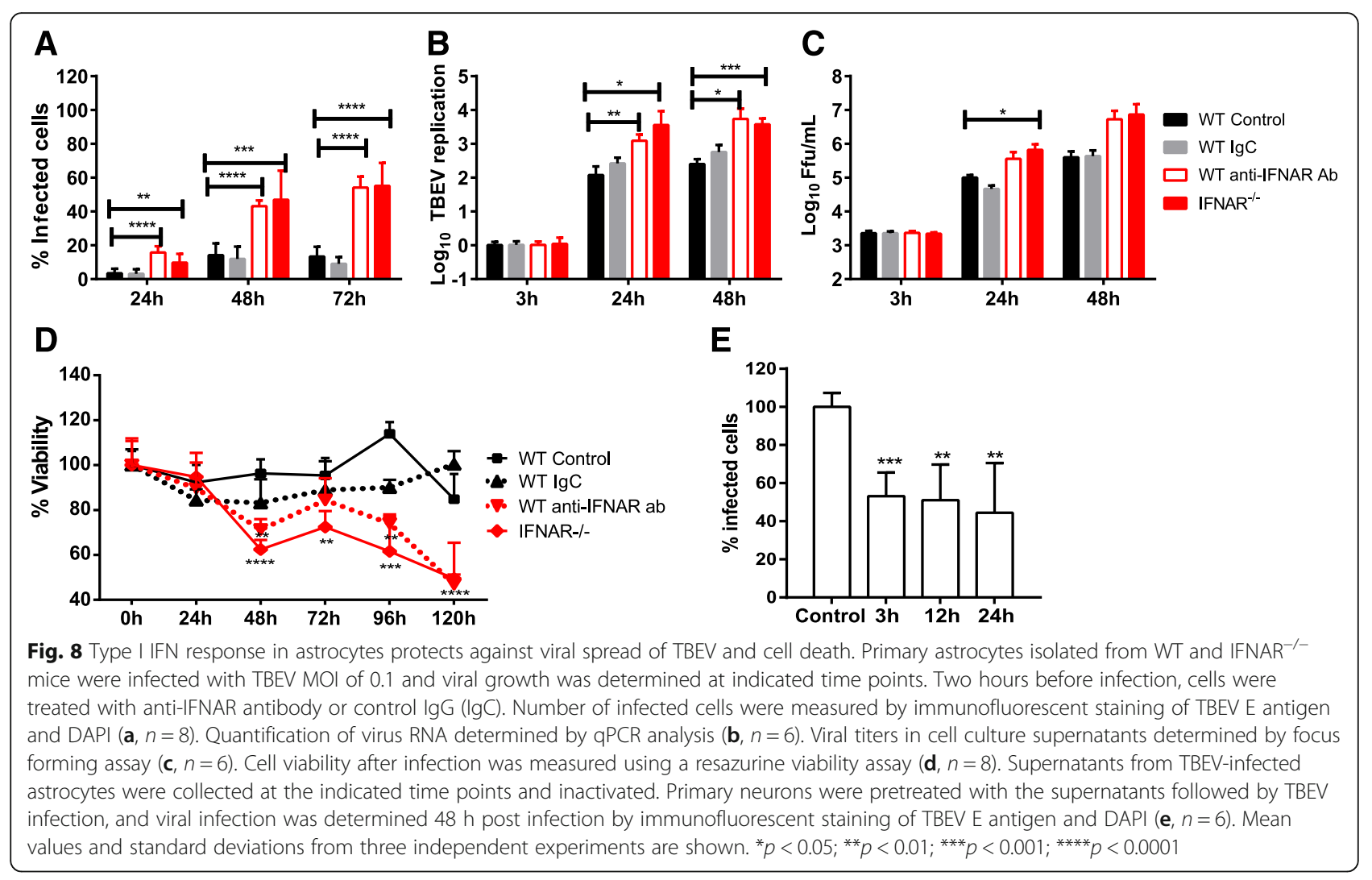

Weak IFN signals, transmitted independently of viral infection, could be crucial for predisposing cells to amplify their IFN production in response to viral infection and enhance their response to other cytokines [74, 75]. Intriguingly, WT astrocytes were in an antiviral state with higher levels of ISGs compared to MEFs, and these were rapidly induced to even higher levels after TBEV infection. Because no difference in basal levels of IFN $\beta$ or IFN $\alpha 2$ was observed between MEFs and WT astrocytes (data not shown), a different mechanism compared to IFN priming may exist [76]. However, it seems that the responsiveness to viral infection is determined by basal levels of innate immune components. We have reported previously that TBEV is able to delay IFN $\beta$ induction by hiding its dsRNA within replication vesicles in A549 cells [42, 77] and a similar observation was made in the MEFs in this study. No delay in type I IFN induction was observed in astrocytes, clearly indicating that if certain antiviral factors are present in the cell at a basal level the virus is unable to delay the IFN response. High basal expression of ISGs have also been linked to viral resistance to influenza A infection in bronchial epithelial cells [78] and contribute to neuronal tropism of WNV [19]. Furthermore, we previously showed that viperin is a strong inhibitor of TBEV infection [50]. Therefore, the increased basal expression of some key ISGs could contribute to lower flavivirus replication in astrocytes. However, the basal expression was not enough by itself to restrict TBEV replication or spread as neutralization of IFNAR with antibodies in WT astrocytes rendered the WT cells susceptible to TBEV infection. Virus infection has been shown to directly induce antiviral ISGs independently of IFN signaling [79]; however, in the case of TBEV infection of astrocytes, this response does not seem to inhibit TBEV growth.

Although IFNAR ${ }^{-/}$astrocytes did induce ISGs at the mRNA level and viperin at the protein level, the kinetics were delayed compared to WT astrocytes indicating the importance of a rapid IFN response in order to control TBEV infection. To further characterize what makes the bystander cells resistant to flavivirus infection, astrocytes were treated with either inactivated supernatant from virus-infected cells or with IFN $\alpha B / D$ followed by RNAseq. The RNAseq revealed that IFN signaling was the most upregulated pathway in supernatant-treated cells suggesting the IFN might be the most important signaling molecule produced by astrocytes upon TBEV infection. This is also true for other viruses, as increased expression of IFN signaling molecules and ISGs was observed in human astrocytes infected with Junin virus [52].

Comparison of the differently expressed genes among WT, IFNAR $^{-/-}$astrocytes, supernatant or IFN $\alpha B / D$-treated WT astrocytes showed an overlap of 112 transcripts, and these transcripts might be of particular importance as most 
of them were downregulated in IFNAR ${ }^{-1-}$ astrocytes, which were highly susceptible to TBEV infection, whereas they were upregulated in supernatant- and IFN $\alpha B / D$-treated cells, which were resistant to the infection. Several antiviral ISGs were identified among the overlap such as viperin and TRIM79 $\alpha$, which have been identified as inhibitors of TBEV [50, 61]. ISG15, viperin, and Oas1b, which were found to be downregulated in IFNAR ${ }^{-/-}$astrocytes and upregulated after treatment with IFN $\alpha \mathrm{B} / \mathrm{D}$ and supernatant, have previously been identified as inhibitors of WNV and could thus contribute to antiviral response against WNV in astrocytes [19, 62, 80, 81].

Predicting upstream regulators responsible for the different expression patterns in IFNAR ${ }^{-/-}$-, supernatant-, and IFN $\alpha B / D$-treated astrocytes identified type I IFN as well as IFN signaling molecules to have the highest activation scores. Similar findings were found in a previous study where IFN signaling and antiviral mediators were among the most upregulated pathways and genes in WNV-infected mice brains [51]. IFNY-STAT1-IRF-1 signaling cascade was predicted as an upstream regulator both in supernatant and IFN $\alpha B / D$-treated astrocytes. Although IFN $\gamma$ transcripts were not detected in TBEV infected, astrocytes IRF-1 could be directly induced by virus infection and could be responsible for the induction of the overlap between inducible genes among the type I and II IFNs [79, 82-84]. Taken together, the RNAseq confirms the potent IFN response of the astrocytes and identifies a subset of genes as key players in determining the outcome of TBEV infection in astrocytes.

Previous studies have revealed that although TBEV infects astrocytes, viral infection did not affect the viability of the cells $[34,35]$. Similar findings have been observed with other viral infections such as WNV, JEV, and Junin virus whereas infection with Venezuelan equine encephalitis virus infection induced cell death in cultured astrocytes [31, 37, 39, 85, 86]. However, the mechanism underlying the resistance to virus-induced cell death in astrocytes is not well understood. Here, we show that the type I IFN response prevents TBEV-mediated cytopathic effect. Blocking of IFNAR using antibodies as well as IFNAR knockout induced TBEV-mediated cell death of astrocytes whereas the WT control remained unaffected.

\section{Conclusions}

Our results show that astrocytes mount a rapid type I IFN response upon flavivirus infection that inhibits viral spread and replication. The IFN response also protects astrocytes from virus-induced cell death. We further propose that astrocytes are important IFN producers in flavivirus infection, which can sense the viral infection and then mediate a local IFN response within the CNS, which could protect not only astrocytes but also other CNS resident cells.

\section{Additional file}

Additional file 1: Supplemental Table S1, Table S2, Table S3, and Table S4. (DOC $210 \mathrm{~kb}$ )

\begin{abstract}
Abbreviations
CMAP: Connectivity Map; dsRNA: Double-stranded RNA; GFAP: Glial fibrillary acidic protein; GSEA: Gene set enrichment analysis; hpi: Hours post infection; IFN: Interferon; IFNAR: Interferon alpha receptor; IPA: Ingenuity Pathway Analysis; IPS-1: Interferon-beta promoter stimulator 1; IRF: Interferon regulatory factor; ISG: Interferon-stimulated gene; ISGF3: Interferon-stimulated gene factor-3; JEV: Japanese encephalitis virus; LGTV: Langat virus; MEF: Mouse embryonic fibroblast; NES: Normalized enrichment score; PRR: Pattern recognition receptor; qPCR: Quantitative reverse transcription PCR; RNAseq: RNA sequencing; STAT: Signal transducer and activator of transcription; TBEV: Tick-borne encephalitis virus; TRIM79a: Tripartite motif 79 a; VSV: Vesicular stomatitis virus; WNV: West Nile virus; WT: Wild-type; ZIKV: Zika virus; $\beta-P L$ : Beta propiolactone
\end{abstract}

\section{Acknowledgements}

We thank Gerhard Dobler from the Bundeswehr Institute of Microbiology (Munich, Germany) for providing the virus strains TBEV strain Hypr 71, Aina, and Sofjin and Sirkka Vene for providing JEV and WNV.

\section{Funding}

This work was supported by the Kempe Foundations, the Laboratory for Molecular Medicine Sweden (MIMS), the Umeå Center for Microbial Research (UCMR) and Linneus Support, and the Swedish Foundation for International Cooperation in Research and Higher Education (STINT), Swedish Research Council (AKÖ)

Availability of data and materials

All data and materials are available upon request.

\section{Authors' contributions}

$\mathrm{RL}$ designed and performed the experiments; analyzed, compiled, and interpreted the data; and wrote the manuscript. FM did all the bioinformatics analysis and wrote the manuscript. JDG, SW, and NOG provided unique expertise and reagents and critically revised the manuscript. AK helped with the design of the study and critically revised the manuscript. AKÖ designed, interpreted, and compiled the data; wrote the manuscript; and provided funding. All authors read and approved the final manuscript.

\section{Competing interests}

The authors declare that they have no competing interests.

\section{Consent for publication}

Not applicable.

\section{Ethics approval}

Animal experiments were approved and performed according to the guidelines set out by the Regional Animal Ethic Committee approval no A77-14, Umeå, Sweden.

\section{Author details}

'Department of Clinical Microbiology, Virology, Umeå University, 90185 Umeå, Sweden. ${ }^{2}$ The Laboratory for Molecular Infection Medicine Sweden (MIMS), 90187 Umeå, Sweden. ${ }^{3}$ The Broad Institute of MIT and Harvard, Proteomics and Biomarkers, 415 Main Street, \#5033-A, Cambridge, MA 02142, USA. ${ }^{4}$ Department of Pharmacology and Clinical Neuroscience, Umeå University, 90187 Umeå, Sweden. ${ }^{5}$ Bundeswehr Institute of Microbiology, Neuherbergstraße 11, 80937 Munich, Germany. ${ }^{6}$ Department of Molecular Biology, Umeå University, 90187 Umeå, Sweden. ${ }^{7}$ Innate Immunity and Infection, Helmholtz Centre for Infection Research, Inhoffen Str 7, 38124 Braunschweig, Germany. ${ }^{8}$ Institute of Medical Microbiology,

Otto-von-Guericke-University Magdeburg, Leipziger Str. 44, 39120 Magdeburg, Germany.

Received: 9 August 2016 Accepted: 16 October 2016 Published online: 24 October 2016 


\section{References}

1. Mansfield KL, Johnson N, Phipps LP, Stephenson JR, Fooks AR, Solomon T. Tick-borne encephalitis virus - a review of an emerging zoonosis. J Gen Virol. 2009;90:1781-94

2. Dumpis U, Crook D, Oksi J. Tick-borne encephalitis. Clin Infect Dis. 1999;28:882-90.

3. Beasley DW. Vaccines and immunotherapeutics for the prevention and treatment of infections with West Nile virus. Immunotherapy. 2011;3:269-85.

4. Diamond MS. Progress on the development of therapeutics against West Nile virus. Antiviral Res. 2009;83:214-27.

5. Bossi P, Tegnell A, Baka A, Van Loock F, Hendriks J, Werner A, Maidhof H, Gouvras G, Task Force on b, Chemial Agent Threats PHDECL. Bichat guidelines for the clinical management of viral encephalitis and bioterrorism-related viral encephalitis. Euro Surveill. 2004;9:E21-2.

6. Tiroumourougane SV, Raghava P, Srinivasan S. Japanese viral encephalitis. Postgrad Med J. 2002;78:205-15.

7. Stetson DB, Medzhitov R. Type I interferons in host defense. Immunity 2006;25:373-81.

8. Takeuchi O, Akira S. Innate immunity to virus infection. Immunol Rev. 2009;227:75-86

9. Gonzalez-Navajas JM, Lee J, David M, Raz E. Immunomodulatory functions of type I interferons. Nat Rev Immunol. 2012;12:125-35.

10. Platanias LC. Mechanisms of type-l- and type-II-interferon-mediated signalling. Nat Rev Immunol. 2005;5:375-86.

11. Weber E, Finsterbusch K, Lindquist R, Nair S, Lienenklaus S, Gekara NO, Janik $D$, Weiss $S$, Kalinke U, Overby AK, Kroger A. Type I interferon protects mice from fatal neurotropic infection with Langat virus by systemic and local antiviral responses. J Virol. 2014;88:12202-12.

12. Lazear HM, Pinto AK, Vogt MR, Gale Jr M, Diamond MS. Beta interferon controls West Nile virus infection and pathogenesis in mice. J Virol. 2011:85:7186-94.

13. Samuel MA, Diamond MS. Alpha/beta interferon protects against lethal West Nile virus infection by restricting cellular tropism and enhancing neuronal survival. J Virol. 2005;79:13350-61.

14. Aoki K, Shimada S, Simantini DS, Tun MM, Buerano CC, Morita K, Hayasaka D. Type-I interferon response affects an inoculation dose-independent mortality in mice following Japanese encephalitis virus infection. Virol J. 2014;11:105

15. Lazear HM, Govero J, Smith AM, Platt DJ, Fernandez E, Miner J, Diamond MS. A mouse model of Zika virus pathogenesis. Cell Host Microbe. 2016:19:720-30.

16. Harris MG, Hulseberg P, Ling C, Karman J, Clarkson BD, Harding JS, Zhang M, Sandor A, Christensen K, Nagy A, et al. Immune privilege of the CNS is not the consequence of limited antigen sampling. Sci Rep. 2014;4:4422.

17. Nair S, Michaelsen-Preusse K, Finsterbusch K, Stegemann-Koniszewski S, Bruder D, Grashoff M, Korte M, Koster M, Kalinke U, Hauser H, Kroger A Interferon regulatory factor-1 protects from fatal neurotropic infection with vesicular stomatitis virus by specific inhibition of viral replication in neurons. PLoS Pathog. 2014;10, e1003999.

18. Detje CN, Lienenklaus S, Chhatbar C, Spanier J, Prajeeth CK, Soldner C, Tovey MG, Schluter D, Weiss S, Stangel M, Kalinke U. Upon intranasal vesicular stomatitis virus infection, astrocytes in the olfactory bulb are important interferon beta producers that protect from lethal encephalitis. J Virol. 2015:89:2731-8.

19. Cho H, Proll SC, Szretter KJ, Katze MG, Gale Jr M, Diamond MS. Differential innate immune response programs in neuronal subtypes determine susceptibility to infection in the brain by positive-stranded RNA viruses. Nat Med. 2013;19:458-64.

20. Gelpi E, Preusser M, Garzuly F, Holzmann H, Heinz FX, Budka H. Visualization of Central European tick-borne encephalitis infection in fatal human cases. J Neuropathol Exp Neurol. 2005;64:506-12.

21. Cho H, Shrestha B, Sen GC, Diamond MS. A role for Ifit2 in restricting West Nile virus infection in the brain. J Virol. 2013;87:8363-71.

22. Reinert LS, Harder L, Holm CK, Iversen MB, Horan KA, Dagnaes-Hansen F, Ulhoi BP, Holm TH, Mogensen TH, Owens T, et al. TLR3 deficiency renders astrocytes permissive to herpes simplex virus infection and facilitates establishment of CNS infection in mice. J Clin Invest. 2012;122:1368-76.

23. Kallfass C, Ackerman A, Lienenklaus S, Weiss S, Heimrich B, Staeheli P. Visualizing production of beta interferon by astrocytes and microglia in brain of La Crosse virus-infected mice. J Virol. 2012;86:11223-30.

24. Lothman EW, Somjen GG. Extracellular potassium activity, intracellular and extracellular potential responses in the spinal cord. J Physiol. 1975;252:115-36.
25. Kimelberg HK, Biddlecome S, Bourke RS. SITS-inhibitable Cl- transport and $\mathrm{Na}+$-dependent $\mathrm{H}+$ production in primary astroglial cultures. Brain Res. 1979;173:111-24.

26. Martinez-Hernandez A, Bell KP, Norenberg MD. Glutamine synthetase: glial localization in brain. Science. 1977;195:1356-8.

27. Aschner M. Neuron-astrocyte interactions: implications for cellular energetics and antioxidant levels. Neurotoxicology. 2000;21:1101-7.

28. Farina C, Aloisi F, Meinl E. Astrocytes are active players in cerebral innate immunity. Trends Immunol. 2007;28:138-45.

29. Kornyey S. Contribution to the histology of tick-borne encephalitis. Acta Neuropathol. 1978;43:179-83.

30. German AC, Myint KS, Mai NT, Pomeroy I, Phu NH, Tzartos J, Winter P, Collett J, Farrar J, Barrett A, et al. A preliminary neuropathological study of Japanese encephalitis in humans and a mouse model. Trans R Soc Trop Med Hyg. 2006:100:1135-45.

31. van Marle G, Antony J, Ostermann H, Dunham C, Hunt T, Halliday W, Maingat F, Urbanowski MD, Hobman T, Peeling J, Power C. West Nile virusinduced neuroinflammation: glial infection and capsid protein-mediated neurovirulence. J Virol. 2007:81:10933-49.

32. Sips GJ, Wilschut J, Smit JM. Neuroinvasive flavivirus infections. Rev Med Virol. 2012;22:69-87.

33. Kurhade C, Zegenhagen L, Weber E, Nair S, Michaelsen-Preusse K, Spanier J, Gekara NO, Kroger A, Overby AK. Type I interferon response in olfactory bulb, the site of tick-borne flavivirus accumulation, is primarily regulated by IPS-1. J Neuroinflammation. 2016;13:22.

34. Palus M, Bily T, Elsterova J, Langhansova H, Salat J, Vancova M, Ruzek D. Infection and injury of human astrocytes by tick-borne encephalitis virus. J Gen Virol. 2014:95:2411-26.

35. Potokar M, Korva M, Jorgacevski J, Avsic-Zupanc T, Zorec R. Tick-borne encephalitis virus infects rat astrocytes but does not affect their viability. PLoS One. 2014;9, e86219.

36. Hussmann KL, Samuel MA, Kim KS, Diamond MS, Fredericksen BL. Differential replication of pathogenic and nonpathogenic strains of West Nile virus within astrocytes. J Virol. 2013;87:2814-22.

37. Diniz JA, Da Rosa AP, Guzman H, Xu F, Xiao SY, Popov VL, Vasconcelos PF, Tesh RB. West Nile virus infection of primary mouse neuronal and neuroglial cells: the role of astrocytes in chronic infection. Am J Trop Med Hyg. 2006:75:691-6.

38. Lucas M, Frenkiel MP, Mashimo T, Guenet JL, Deubel V, Despres P, Ceccaldi PE. The Israeli strain IS-98-ST1 of West Nile virus as viral model for West Nile encephalitis in the Old World. Virol J. 2004;1:9.

39. Chang CY, Li JR, Chen WY, Ou YC, Lai CY, Hu YH, Wu CC, Chang CJ, Chen $\mathrm{CJ}$. Disruption of in vitro endothelial barrier integrity by Japanese encephalitis virus-infected astrocytes. Glia. 2015;63:1915-932.

40. Schildge S, Bohrer C, Beck K, Schachtrup C. Isolation and culture of mouse cortical astrocytes. J Vis Exp. 2013;e50079:1-7.

41. Dunning CJ, MCGauran G, Willen K, Gouras GK, O'Connell DJ, Linse S. Direct high affinity interaction between Abeta42 and GSK3alpha stimulates hyperphosphorylation of tau. A new molecular link in Alzheimer's disease? ACS Chem Neurosci. 2016;7:161-70

42. Overby AK, Popov VL, Niedrig M, Weber F. Tick-borne encephalitis virus delays interferon induction and hides its double-stranded RNA in intracellular membrane vesicles. J Virol. 2010;84:8470-83.

43. Schwaiger M, Cassinotti P. Development of a quantitative real-time RT-PCR assay with internal control for the laboratory detection of tick borne encephalitis virus (TBEV) RNA. J Clin Virol. 2003;27:136-45.

44. Niedrig M, Klockmann U, Lang W, Roeder J, Burk S, Modrow S, Pauli G. Monoclonal antibodies directed against tick-borne encephalitis virus with neutralizing activity in vivo. Acta Virol. 1994;38:141-9.

45. Cargnelutti JF, Brum MC, Weiblen R, Flores EF. Stable expression and potential use of West Nile virus envelope glycoproteins preM/E as antigen in diagnostic tests. Braz J Microbiol. 2011;42:1161-6.

46. Yousef H, Conboy MJ, Morgenthaler A, Schlesinger C, Bugaj L, Paliwal P, Greer C, Conboy IM, Schaffer D. Systemic attenuation of the TGF-beta pathway by a single drug simultaneously rejuvenates hippocampal neurogenesis and myogenesis in the same old mammal. Oncotarget. 2015:6:11959-78.

47. Perler $L$, Pfister $H$, Schweizer M, Peterhans E, Jungi TW. A bioassay for interferon type I based on inhibition of Sendai virus growth. J Immunol Methods. 1999:222:189-96.

48. Barrett AD, Hunt N, Dimmock NJ. A rapid method for the inactivation of virus infectivity prior to assay for interferons. J Virol Methods. 1984;8:349-51. 
49. Horisberger MA, de Staritzky K. A recombinant human interferon-alpha B/D hybrid with a broad host-range. J Gen Virol. 1987;68(Pt 3):945-8.

50. Upadhyay AS, Vonderstein K, Pichlmair A, Stehling O, Bennett KL, Dobler G, Guo JT, Superti-Furga G, Lill R, Overby AK, Weber F. Viperin is an iron-sulfur protein that inhibits genome synthesis of tick-borne encephalitis virus via radical SAM domain activity. Cell Microbiol. 2014;16:834-48.

51. Clarke P, Leser JS, Quick ED, Dionne KR, Beckham JD, Tyler KL. Death receptor-mediated apoptotic signaling is activated in the brain following infection with West Nile virus in the absence of a peripheral immune response. J Virol. 2014;88:1080-9.

52. Kolokoltsova OA, Yun NE, Paessler S. Reactive astrogliosis in response to hemorrhagic fever virus: microarray profile of Junin virus-infected human astrocytes. Virol J. 2014;11:126.

53. Subramanian A, Tamayo P, Mootha VK, Mukherjee S, Ebert BL, Gillette MA, Paulovich A, Pomeroy SL, Golub TR, Lander ES, Mesirov JP. Gene set enrichment analysis: a knowledge-based approach for interpreting genomewide expression profiles. Proc Natl Acad Sci U S A. 2005;102:15545-50.

54. Lamb J, Crawford ED, Peck D, Modell JW, Blat IC, Wrobel MJ, Lerner J, Brunet JP, Subramanian A, Ross KN, et al. The connectivity map: using geneexpression signatures to connect small molecules, genes, and disease. Science. 2006:313:1929-35.

55. Trapnell C, Roberts A, Goff L, Pertea G, Kim D, Kelley DR, Pimentel H, Salzberg SL, Rinn JL, Pachter L. Differential gene and transcript expression analysis of RNA-seq experiments with TopHat and Cufflinks. Nat Protoc. 2012;7:562-78

56. Trapnell C, Williams BA, Pertea G, Mortazavi A, Kwan G, van Baren MJ, Salzberg SL, Wold BJ, Pachter L. Transcript assembly and quantification by RNA-Seq reveals unannotated transcripts and isoform switching during cell differentiation. Nat Biotechnol. 2010;28:511-5.

57. Gritsun TS, Frolova TV, Zhankov Al, Armesto M, Turner SL, Frolova MP, Pogodina W, Lashkevich VA, Gould EA. Characterization of a Siberian virus isolated from a patient with progressive chronic tick-borne encephalitis. J Virol. 2003;77:25-36.

58. Dorrbecker B, Dobler G, Spiegel M, Hufert FT. Tick-borne encephalitis virus and the immune response of the mammalian host. Travel Med Infect Dis. 2010:8:213-22

59. Hamel R, Dejarnac O, Wichit S, Ekchariyawat P, Neyret A, Luplertlop N, Perera-Lecoin M, Surasombatpattana P, Talignani L, Thomas F, et al. Biology of Zika virus infection in human skin cells. J Virol. 2015;89:8880-96.

60. Aliota MT, Caine EA, Walker EC, Larkin KE, Camacho E, Osorio JE. Characterization of lethal Zika virus infection in AG129 mice. PLoS Negl Trop Dis. 2016;10, e0004682

61. Taylor RT, Lubick KJ, Robertson SJ, Broughton JP, Bloom ME, Bresnahan WA, Best SM. TRIM79alpha, an interferon-stimulated gene product, restricts tickborne encephalitis virus replication by degrading the viral RNA polymerase. Cell Host Microbe. 2011;10:185-96.

62. Szretter KJ, Brien JD, Thackray LB, Virgin HW, Cresswell P, Diamond MS. The interferon-inducible gene viperin restricts West Nile virus pathogenesis. J Virol. 2011;85:11557-66.

63. Sheehan KC, Lai KS, Dunn GP, Bruce AT, Diamond MS, Heutel JD, DungoArthur C, Carrero JA, White JM, Hertzog PJ, Schreiber RD. Blocking monoclonal antibodies specific for mouse IFN-alpha/beta receptor subunit 1 (IFNAR-1) from mice immunized by in vivo hydrodynamic transfection. J Interferon Cytokine Res. 2006;26:804-19.

64. Kurhade C, Zegenhagen L, Weber E, Nair S, Michaelsen-Preusse K, Spanier J, Gekara NO, Kröger A, Överby AK. Type I interferon response in olfactory bulb, the site of tick-borne flavivirus accumulation, is primarily regulated by IPS-1. J Neuroinflammation. 2016;13.

65. Griffin DE. Immune responses to RNA-virus infections of the CNS. Nat Rev Immunol. 2003:3:493-502.

66. Muller U, Steinhoff U, Reis LF, Hemmi S, Pavlovic J, Zinkernagel RM, Aguet M. Functional role of type I and type II interferons in antiviral defense. Science. 1994;264:1918-21.

67. Ryman KD, Klimstra WB, Nguyen KB, Biron CA, Johnston RE. Alpha/beta interferon protects adult mice from fatal Sindbis virus infection and is an important determinant of cell and tissue tropism. J Virol. 2000;74:3366-78.

68. Detje CN, Meyer T, Schmidt H, Kreuz D, Rose JK, Bechmann I, Prinz M, Kalinke U. Local type I IFN receptor signaling protects against virus spread within the central nervous system. J Immunol. 2009;182:2297-304.

69. De Miranda J, Yaddanapudi K, Hornig M, Lipkin Wl. Astrocytes recognize intracellular polyinosinic-polycytidylic acid via MDA-5. FASEB J. 2009;23:1064-71.
70. Lieberman AP, Pitha PM, Shin HS, Shin ML. Production of tumor necrosis factor and other cytokines by astrocytes stimulated with lipopolysaccharide or a neurotropic virus. Proc Natl Acad Sci U S A. 1989;86:6348-52.

71. Butchi NB, Du M, Peterson KE. Interactions between TLR7 and TLR9 agonists and receptors regulate innate immune responses by astrocytes and microglia. Glia. 2010;58:650-64.

72. Rivieccio MA, Suh HS, Zhao Y, Zhao ML, Chin KC, Lee SC, Brosnan CF. TLR3 ligation activates an antiviral response in human fetal astrocytes: a role for viperin/cig5. J Immunol. 2006;177:4735-41.

73. Pfefferkorn C, Kallfass C, Lienenklaus S, Spanier J, Kalinke U, Rieder M, Conzelmann KK, Michiels T, Staeheli P. Abortively infected astrocytes appear to represent the main source of interferon beta in the virus-infected brain. J Virol. 2015;90:2031-8.

74. Taniguchi T, Takaoka A. A weak signal for strong responses: interferonalpha/beta revisited. Nat Rev Mol Cell Biol. 2001:2:378-86.

75. Lienenklaus S, Cornitescu M, Zietara N, Lyszkiewicz M, Gekara N, Jablonska J, Edenhofer F, Rajewsky K, Bruder D, Hafner M, et al. Novel reporter mouse reveals constitutive and inflammatory expression of IFN-beta in vivo. J Immunol. 2009;183:3229-36.

76. Kuri T, Zhang X, Habjan M, Martinez-Sobrido L, Garcia-Sastre A, Yuan Z, Weber F. Interferon priming enables cells to partially overturn the SARS coronavirusinduced block in innate immune activation. J Gen Virol. 2009:90:2686-94.

77. Overby AK, Weber F. Hiding from intracellular pattern recognition receptors, a passive strategy of flavivirus immune evasion. Virulence. 2011;2:238-40.

78. Seng LG, Daly J, Chang KC, Kuchipudi SV. High basal expression of interferon-stimulated genes in human bronchial epithelial (BEAS-2B) cells contributes to influenza A virus resistance. PLoS One. 2014;9, e109023.

79. Stirnweiss A, Ksienzyk A, Klages K, Rand U, Grashoff M, Hauser H, Kroger A. IFN regulatory factor-1 bypasses IFN-mediated antiviral effects through viperin gene induction. J Immunol. 2010;184:5179-85.

80. Dai J, Pan W, Wang P. ISG15 facilitates cellular antiviral response to dengue and west Nile virus infection in vitro. Virol J. 2011;8:468.

81. Kajaste-Rudnitski A, Mashimo T, Frenkiel MP, Guenet JL, Lucas M, Despres P. The 2',5'-oligoadenylate synthetase $1 \mathrm{~b}$ is a potent inhibitor of West Nile virus replication inside infected cells. J Biol Chem. 2006:281:4624-37.

82. Sanda C, Weitzel P, Tsukahara T, Schaley J, Edenberg HJ, Stephens MA, McClintick JN, Blatt LM, Li L, Brodsky L, Taylor MW. Differential gene induction by type I and type II interferons and their combination. J Interferon Cytokine Res. 2006;26:462-72.

83. Metz P, Dazert E, Ruggieri A, Mazur J, Kaderali L, Kaul A, Zeuge U, Windisch MP, Trippler M, Lohmann V, et al. Identification of type I and type II interferon-induced effectors controlling hepatitis $C$ virus replication. Hepatology. 2012;56:2082-93.

84. Crotta S, Davidson S, Mahlakoiv T, Desmet CJ, Buckwalter MR, Albert ML, Staeheli P, Wack A. Type I and type III interferons drive redundant amplification loops to induce a transcriptional signature in influenzainfected airway epithelia. PLoS Pathog. 2013;9, e1003773.

85. Pozner RG, Collado S, Jaquenod de Giusti C, Ure AE, Biedma ME, Romanowski V, Schattner M, Gomez RM. Astrocyte response to Junin virus infection. Neurosci Lett. 2008:445:31-5.

86. Schoneboom BA, Fultz MJ, Miller TH, McKinney LC, Grieder FB. Astrocytes as targets for Venezuelan equine encephalitis virus infection. J Neurovirol. 1999:5:342-54.

\section{Submit your next manuscript to BioMed Central and we will help you at every step:}

- We accept pre-submission inquiries

- Our selector tool helps you to find the most relevant journal

- We provide round the clock customer support

- Convenient online submission

- Thorough peer review

- Inclusion in PubMed and all major indexing services

- Maximum visibility for your research

Submit your manuscript at www.biomedcentral.com/submit 